\title{
Complex ambiguity-free Fourier domain optical coherence tomography through transverse scanning
}

\author{
Rainer A. Leitgeb,* Roland Michaely, Theo Lasser, and S. Chandra Sekhar \\ Institute of Applied Optics, Ecole Polytechnique Federale de Lausanne, CH-1015 Lausanne, Switzerland \\ *Corresponding author: rainer.leitgeb@epfl.ch
}

Received October 1, 2007; accepted October 19, 2007;

posted November 6, 2007 (Doc. ID 88113); published November 28, 2007

\begin{abstract}
We introduce a simple and cheap method for phase-shifting Fourier domain optical coherence tomography (FDOCT) that does not need additional devices and can easily be implemented. A small beam offset at the fast beam-scanning mirror introduces a causal phase shift, which can be used for B-scan-based complex image reconstruction. We derive the conditions for optimal conjugate suppression and demonstrate the method on human skin in vivo for spectrometer-based FDOCT operating at $1300 \mathrm{~nm}$ employing a handheld scanner. (C) 2007 Optical Society of America

OCIS codes: $110.4500,170.4500,170.0170$.
\end{abstract}

Employing phase-shifting techniques in Fourier domain optical coherence tomography (FDOCT) is a common method to suppress complex ambiguity terms due to the signal reconstruction. The terms are a direct result of taking the Fourier transform of the recorded spectral interference pattern, which is a real-valued function. The first attempts to produce the complex FDOCT signal were phase stepping techniques known from white-light interferometry [1,2]. However, keeping a clear phase relation over several frames becomes difficult in the case of in vivo measurements. The reduction to two frames allowed motion artifacts to be kept small [3]. Still, the phaseshifting process is highly chromatic, leading to phase errors and failure of mirror term suppression. The use of frequency shifters allows a complete achromatic heterodyne signal reconstruction [4]. The advantages of complex reconstruction techniques in FDOCT are doubling of the achievable depth range, the suppression of mirror terms that might obscure the sample structure, and the possibility of exploiting the high sensitivity across the zero delay. For clinical systems the suppression of mirror terms-avoiding folding of structure terms at the zero delayincreases instrument quality and facilitates the system operation. The drawback of employing phaseshifting devices such as electro-optic modulators, acousto-optic frequency shifters, or piezo transducers is the increased complexity of the system concerning synchronization electronics as well as the price of the elements themselves. In particular for common-path configurations [5], where the reference arm is included in a fiber-coupled hand-held applicator, it is difficult to include such phase-shifting devices. In the present Letter we will show how to realize a simple FDOCT system that allows complex signal reconstruction without additional phase-shifting devices.

It is already known from en face time domain OCT that a heterodyne frequency can be obtained by simply offsetting the probing beam from the pivot point of the scanning mirror [6]. Consider the schematics in Fig. 1(a): the offset $\Delta x$ from the center of rotation (CR) of the scanner mirror causes a change in optical path length $\delta z$ during scanning. For small angles of rotation $\delta \alpha$ the path length change reads as $\delta z$ $=2 \delta \alpha \Delta x /(1-\delta \alpha)$. Of particular interest is the differential path length change between successive depth scans, i.e., during the detection line period $T$. With the relation between the angular scanner frequency $\omega$ and the differential angular change $\Delta(\delta \alpha)$, $\omega=\Delta(\delta \alpha) / T$, we have $\Delta(\delta z)=2 \omega T \Delta x /(1-\delta \alpha)^{2}$ $\left.\approx 2 \omega T \Delta x\right|_{\delta \alpha=0}$. For small angles around $\delta \alpha=0$ we find a linear relation between path length change and scanner offset, resulting in a phase difference between successive A scans of $\Delta \phi=4 \pi \Delta(\delta z) / \lambda$ $=8 \omega T \Delta x \pi / \lambda$.

(a)

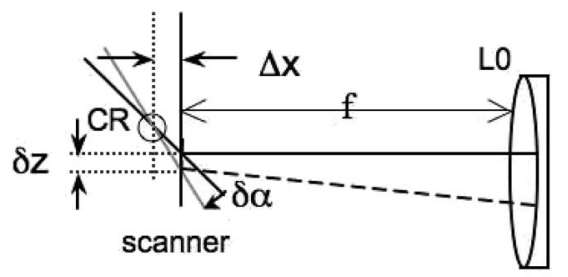

(b)

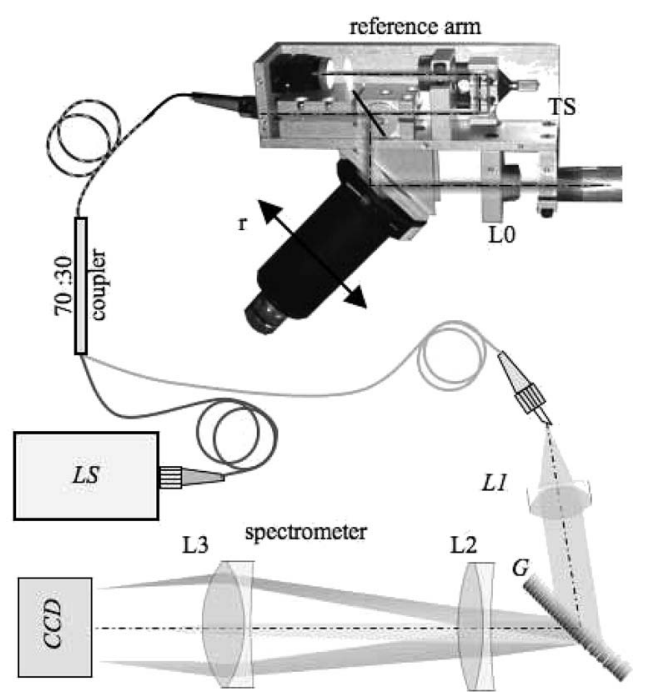

Fig. 1. Schematics of (a) scanner offset geometry and (b) optical setup; see text for details. 
Recently Yasuno et al. [7] published a B-scanbased-method for complex signal reconstruction. They analyzed the phase evolution for each optical frequency across the tomogram or B scan introduced by phase stepping the reference arm delay. Later Wang [8] showed that an arbitrary continuous but causal phase shifting of the reference delay is sufficient for complete complex continuation of the signal with a Hilbert transform (HT). Observing that the phase offset due to the scanner movement will remain causal, i.e., the phase gradient will not change sign during lateral scanning, we can employ the B-scan-based HT reconstruction technique. The complex signal is obtained for a complete tomogram or B-scan via $\widetilde{I}_{k}(x)=I_{k}(x)-i H\left\{I_{k}(x)\right\}$, where the Hilbert transform $H$ is taken along the transverse coordinate $x$ for each wavenumber channel $k$. In a second step the unambiguous depth structure is found via Fourier transform (FT) of the complex valued spectrum along $k$ at each transverse point $x$, i.e., $\widetilde{I}_{x}(z)$ $=\mathrm{FT}\left\{\widetilde{I}_{x}(k)\right\}$. The HT-based analytic continuation of the signal will in theory yield the correct complex signal no matter the actual phase shift. Still, as we scan laterally we have two quantities that have direct impact on the complex reconstruction: first, phase and amplitude noise and, second, the lateral structure spatial frequency spectrum. The spatial frequency spectrum of the sample is of particular interest for the B-scan-based reconstruction algorithm and needs to be considered in view of the Bedrosian theorem. The latter states that for the Hilbert transform to yield the quadrature signal term the signal spectrum needs to be well separated from the introduced modulation frequency. Consider Fig. 2(a): with a tomogram length of $N$ depth profiles covering a transverse range $x$, the spatial frequency range is limited by $\nu_{x}$ $= \pm N /(2 x)$. For coherent imaging we can assume a characteristic width of the detected spatial frequency

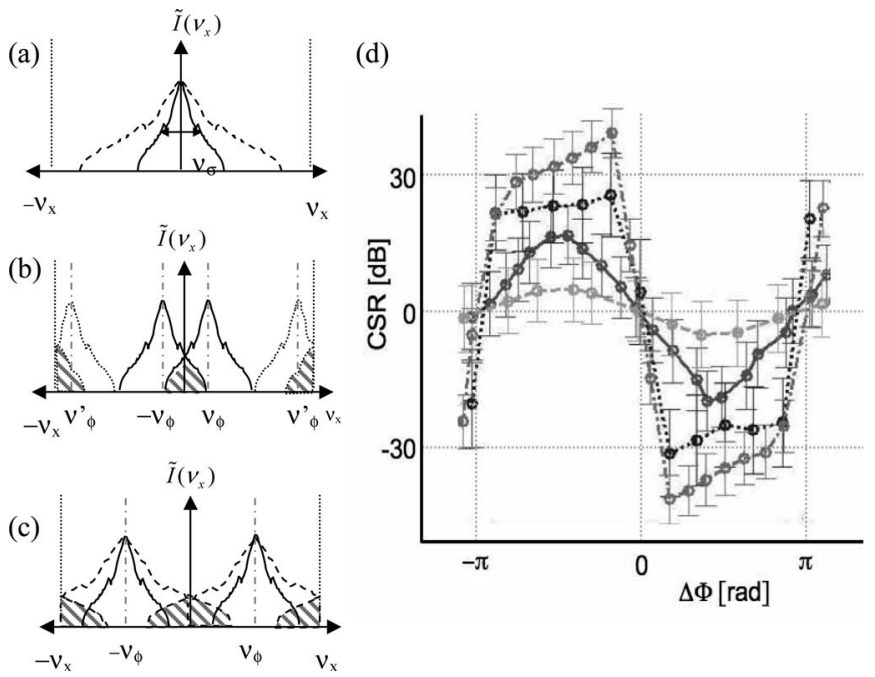

Fig. 2. (a)-(c) Shifting of sample spatial frequency spectrum. (d) Mean CSR over a tomogram depending on phase shift $\Delta \phi$ for a mirror sample (black dotted curve), paper sample scanned at $40 \mathrm{deg} / \mathrm{s}$ with $\rho=5$ (solid curve) and $\rho$ $=40$ (dashed-dotted curve), and with $\rho=5$ but doubled scanning frequency (dashed curve). The error bars are standard deviation values. spectrum to be determined by the inverse speckle size as $\nu_{\sigma}=1 / \sigma$. The speckle size can be written as the transverse resolution, which in the case of Gaussian optics reads as $\sigma=4 \lambda f /(\pi d)$ with $\lambda$ the central wavelength, $f$ the focal length, and $d$ the collimated beam diameter at the object lens. If a modulation is introduced with phase change $\Delta \phi$ between successive depth profiles, the lateral spatial frequency spectrum is shifted by $\pm \nu_{\phi}= \pm(\Delta \phi / 2 \pi)(N / x)$ as indicated in Fig. 2(b). We observe an overlapping region between the two adjointly shifted spectra (shaded region). As the modulation frequency increases, the spectrum will eventually be shifted across the Nyquist boundary, causing aliasing terms to overlap with the spatial spectrum. Within overlap regions the corresponding complex ambiguity terms cannot be resolved. If we express the signal peak amplitude after Fourier transform as $A(z)$ and its mirror conjugate as $A^{*}(-z)$ we define the causing conjugate suppression ratio (CSR) as $|A|^{2} /\left|A^{*}\right|^{2}$. In general the CSR will be reduced in proportion to the spectral overlap area by virtue of Parseval's theorem. The highest CSR is clearly obtained at half the Nyquist frequency corresponding to a phase shift of $\pi / 2$, i.e., $\nu_{\pi / 2}=N /(4 x)$ [Fig. 2(c)]. It is also obvious from Fig. 2(a)-2(d) that the performance of the algorithm strongly depends on the ratio $\rho$ between the spatial spectral width $1 / \sigma$ and the full spectral range $N / x, \rho \equiv N \sigma / x$. If the spatial spectrum is large (dashed spectrum), the optimal CSR will never be achieved because of aliasing terms. In the case of a narrow spatial spectrum in relation to the full spectral range, first, one already has full suppression for moderate modulation frequencies and, second, in the optimal case of Fig. 2(c), even small phase fluctuations will not cause a reduction of the suppression ratio. We therefore deduce the following condition for optimal performance of the HT method:

$$
\nu_{\sigma} \ll \nu_{\pi / 2}=N / 4 x \quad \text { or } \quad N / x \gg \pi d / \lambda f .
$$

Alternatively one can write for the sampling ratio $\rho$ $\gg 4$.

For our proposed phase-shifting method the desired phase shift $\Delta \phi$ of $\pi / 2$ results in the following relation between beam offset on the scanner, acquisition period, and scanning frequency $\omega T \Delta x=\lambda / 16$, where we used the relation from paragraph 2 above for $\Delta \phi$. Since the scanning range $x$ can be expressed as $x=\omega N T f$, from Eq. (1) we arrive at the condition $1 / \omega T \gg \pi d / \lambda$.

For experimental verification of the theory we used the fiber-based common-path FDOCT system depicted in Fig. 1(b). It employs an AFC light source (LS) with a center wavelength at $1300 \mathrm{~nm}$ and a spectral FWHM of $70 \mathrm{~nm}$. The resulting axial resolution is $10 \mu \mathrm{m}$ in air. The 70:30 fiber coupler passes $30 \%$ of the light to the sample arm fiber equipped with a hand-held scanner, which constitutes the adjustable reference arm. Within the hand-held scanner a nonpolarizing beam splitter divides sample and reference arm light. The sample arm beam is deflected by a single-point 2D scanner and focused via a $50 \mathrm{~mm}$ achromat (L0) into the sample. The spectrom- 
eter at the exit of the fiber coupler consists of a transmission grating (G, 1200 lines/mm, Wasatch, Inc.) and a line array (CCD, Sensors Unlimited, 512 pixel, 12 bit). The camera objective is an achromat combination (L2, L3) with an effective focal length of $100 \mathrm{~mm}$. The spectrometer resolution is $0.28 \mathrm{~nm}$, allowing for a system depth range of $1.5 \mathrm{~mm}$. The actual exposure time is $22 \mu \mathrm{s}$ with a frame rate of $18.9 \mathrm{kHz}$ or $T=53 \mu \mathrm{s}$. With a power at the sample of $2 \mathrm{~mW}$ the sensitivity was measured to be $105 \mathrm{~dB}$, close to the zero delay with a signal falloff of $-12 \mathrm{~dB} / \mathrm{mm}$. The beam offset is adjusted via diagonal translation $r$ of the scanner, which keeps the beam at the center of the focusing optics.

In Fig. 2(d) we plotted the measured mean CSR dependence on phase shift $\Delta \phi$. The mean CSR was obtained across a full tomogram after applying an intensity threshold. The associated mean phase difference was calculated across a tomogram by taking the difference of the arguments of successive Fourier transformed complex spectra. The scanning angle was \pm 1 deg optically with a mechanical scanning angular speed of $40 \mathrm{deg} / \mathrm{s}$, resulting in a lateral scanning range of $1.8 \mathrm{~mm}$ sampled by $N=450$ points. For the paper sample we varied the ratio $\rho$ by changing the beam waist $d$. The beam diameter was 4 and $0.5 \mathrm{~mm}$, resulting in spot sizes $\sigma$ of 20 and $160 \mu \mathrm{m}$, respectively, and a ratio $\rho$ of 5 and 40 . The mirror has almost zero spatial frequencies, resulting, as expected, in optimal mirror term suppression as soon as a modulation offset is applied (dotted curve). In the case of large $\sigma$, for the paper sample we also reach the optimal suppression ratio for small offsets (dashed-dotted curve). As we increase the resolution we decrease the spot size $\sigma$ and expect, because of the broader spatial frequency spectrum, suboptimal conjugate term suppression, which is indeed verified by the experiment (solid curve). If we double the scanner speed without changing the sampling $\rho$, we strongly decrease the CSR owing to stronger phase fluctuations [9]. The phase error was $\sim 12 \mathrm{deg}$ and increased to almost $20 \mathrm{deg}$ for fast scanning. Another important observation is the change of sign of the suppression ratio at $m \pi$ for $m=0, \pm 1, \pm 2, \ldots$. As a consequence the structure will be mirrored across the zero delay if the phase shift crosses those points.

Finally we demonstrate the ability of the method to perform complex ambiguity-free in vivo imaging. According to our previous findings we chose a scanner angular speed of $17.5 \mathrm{deg} / \mathrm{s}$ with a tomogram width of 1800 lateral depth scans corresponding to $3 \mathrm{~mm}$ transversally and $\rho=12$ for $\sigma=20 \mu \mathrm{m}$. The scanner offset was adjusted to $4.5 \mathrm{~mm}$ from the pivot point. Figure 3(a) displays a standard reconstructed tomogram of a human inner lip in vivo. The complex mirror terms heavily overlap with the structure. Figures 3(b) and 3(c) display orthogonal cross sections through a $3 \mathrm{D}$ data set consisting of 150 tomograms spanning over $2 \mathrm{~mm}$ in the vertical direction. The displayed depth range is $2 \mathrm{~mm}$. The tomogram series has been registered, and averaging over five tomo-
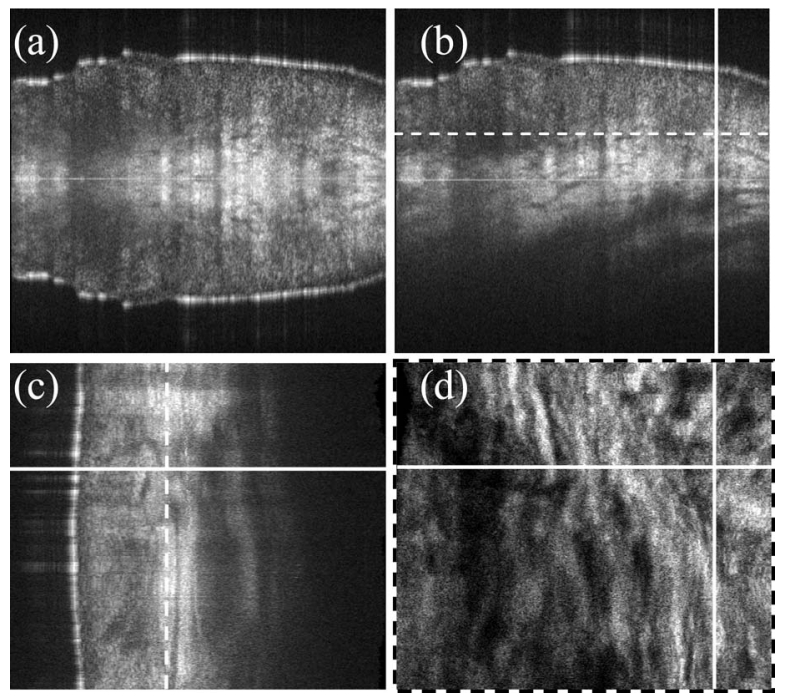

Fig. 3. Tomogram of human inner lip $(1800 \times 512$ pixels, $3 \mathrm{~mm} \times 2.5 \mathrm{~mm}$ ), with (a) standard and (b) complex reconstruction; (c) orthogonal cross section of (b); (d) en face section along dashed lines in (b) and (c) at a skin depth of $\sim 700 \mu \mathrm{m}$.

grams has been applied for speckle reduction. The complex ambiguity terms have been efficiently removed by employing the B-scan based technique, as is seen from Figs. 3(b) and 3(c). The CSR in Fig. 3(b) is better than $30 \mathrm{~dB}$. Figure $3(\mathrm{~d})$ shows an en face slice of the skin volume extracted at a depth of $\sim 700 \mu \mathrm{m}$.

In conclusion, we demonstrated a cheap method for complex ambiguity-suppressed FDOCT imaging and derived the conditions under which optimal performance is achieved. The findings allow straightforward implementation in any FDOCT system, especially for devices with fiber-coupled hand-held applicators.

Financial support from the Swiss National Foundation is acknowledged (project 205321-109704/1).

\section{References}

1. A. F. Fercher, R. Leitgeb, C. K. Hitzenberger, H. Sattmann, and M. Wojtkowski, Proc. SPIE 3564, 173 (1999).

2. M. Wojtkowski, A. Kowalczyk, R. Leitgeb, and A. F. Fercher, Opt. Lett. 27, 1415 (2002).

3. R. A. Leitgeb, C. K. Hitzenberger, A. F. Fercher, and T. Bajraszewski, Opt. Lett. 28, 2201 (2003).

4. A. H. Bachmann, R. A. Leitgeb, and T. Lasser, Opt. Express 14, 1487 (2006).

5. A. H. Bachmann, R. Michaely, T. Lasser, and R. A. Leitgeb, Opt. Express 15, 9254 (2007).

6. A. Bradu, A. Podoleanu, and R. B. Rosen, J. Optoelectron. Adv. Mater. 7, 2913 (2005).

7. Y. Yasuno, S. Makita, T. Endo, G. Aoki, M. Itoh, and T. Yatagai, Appl. Opt. 45, 1861 (2006).

8. R. K. Wang, Appl. Phys. Lett. 90, 054103 (2007).

9. B. H. Park, M. C. Pierce, B. Cense, S. H. Yun, M. Mujat, G. J. Tearney, B. E. Bouma, and J. F. de Boer, Opt. Express 13, 3931 (2005). 